\title{
Dislocation multiplication behavior of AZ61 magnesium alloy during
}

\section{cyclic bending}

\author{
TIAN Tie ${ }^{1, a^{*}}$, YUAN Zhao-cheng ${ }^{1}$, MI Lin $^{2}$ and MAO Xing-zi ${ }^{3}$ \\ ${ }^{1}$ State Key Laboratory of Automotive Simulation and Control, Jilin University, Changchun 130022 \\ China \\ ${ }^{2}$ Key Laboratory of Advanced Manufacturing Technology for Automobile Parts, Ministry of Education, \\ Chongqing University of Technology, Chongqing 400054 China \\ ${ }^{3}$ Chongqing Vehicle Test \& Research Institute Co.,Ltd, National Coach Quality Supervision \& \\ Testing Center, Chongqing 401122,China \\ aemail:tiantie1982622@163.com
}

Key Words: AZ61 magnesium alloy, dislocation, strain amplitude, shear stress, multiplication Abstract. The dislocation multiplication behavior of AZ61 magnesium alloy during cyclic bending vibration was investigated. The results show that with the increase of stain amplitude and shear stress, the process of dislocation multiplication behavior contains dislocation slipping, dislocation multiplication and dislocation extension, and dislocation multiplication phenomenon accords with Frank-Read source mechanism. At last, the mobility dislocation number and the dislocation density in AZ61 magnesium alloy increase significantly with the increase of stain amplitude.

\section{Introduction}

Magnesium alloy is the lightest structural material with excellent damping capacity. The result shows that the dislocation movement and the change of mobility dislocation density (Dislocation length per unit volume) in magnesium alloys significantly affect dislocation damping capacity at room temperature [1-5]. And, further researching on the micro-theory of metal mechanical properties, it shows that dislocation multiplication caused by shear stress is responsible for dislocation movement and the change of mobility dislocation density [6]. Therefore, the process of dislocation multiplication behavior and the change of mobility dislocation density caused by dislocation multiplication are important foundations of researching on the dislocation damping mechanism of magnesium alloys. However, up until recently, very little attention has been paid to study the process of dislocation multiplication in magnesium alloys.

Cite the mechanics of materials as a reference, we can see the shear stress generates in the rectangular beam when the beam is in condition of cyclic bending vibration. This study takes cyclic bending vibration mode of the rectangular beam as the research object, and the aim of this study is to study the process of dislocation multiplication in AZ61 magnesium alloy as the stain amplitude increase.

\section{Experimental procedures}

\section{Magnesium alloy preparation}

Commercial cast AZ61 magnesium alloy (5.5-7.0 wt $\% \mathrm{Al}, 0.5-1.5 \mathrm{wt} \% \mathrm{Zn}, 0.15-0.5 \mathrm{wt} \% \mathrm{Mn}$ ) was used as the material. 


\section{Treatment on magnesium alloy rectangular beam}

AZ61 magnesium alloy rectangular beam was cut via wire electrical discharge machining. The dimension of the magnesium alloy rectangular beam was $500 \mathrm{~mm} \times 15 \mathrm{~mm} \times 7 \mathrm{~mm}$. Then, the rectangular beam was treated via cyclic bending deformation, loaded in the middle of beam length, which is shown in Fig. 1. The loading parameters are shown in Table 1.

Table 1 Loading parameters of the beam

\begin{tabular}{ccc}
\hline strain amplitude & bending cycle & frequency \\
\hline $5 \times 10^{-3}$ & 100 & $2 \mathrm{~Hz}$ \\
\hline
\end{tabular}

\section{Direction \& distribution of shear stress in the beam}

When the beams were in condition of cyclic bending vibration, the direction $\&$ distribution of shear stress in the beam was studied. The dimensions of the rectangular beam $\quad\left(a_{1}=500 \mathrm{~mm}, b_{1}=15 \mathrm{~mm}\right.$, $\mathrm{c}_{1}=7 \mathrm{~mm}$ ) are shown in Fig. 1.

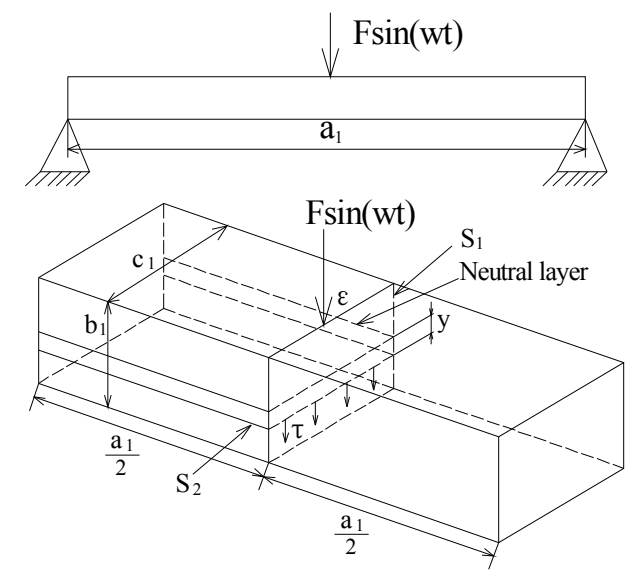

Fig. 1.Dimensions of the rectangular beam and direction $\&$ distribution of shear stress

$\mathrm{S}_{1}$ is the shear stress action face; $\mathrm{S}_{2}$ is the parallel layer with the neutral layer

The direction \& distribution of shear stress $\tau$ in $S_{1}$ layer is shown in Fig. 1 and formula (3)

$$
\begin{aligned}
& \tau=\frac{F \sin (\omega t)\left(b_{1}^{2} / 4-y^{2}\right)}{4 I_{z}} \\
& \varepsilon=\frac{\sigma}{E}=\frac{F \sin (\omega t) a_{1} b_{1}}{8 I_{z} E} \\
& \tau=\frac{2\left(b_{1}^{2} / 4-y^{2}\right) E}{a_{1} b_{1}} \varepsilon
\end{aligned}
$$

Where, $\tau$ is the shear stress value in $\mathrm{S}_{2}$ layer, $\varepsilon$ is strain amplitude, $y$ is the distance of the parallel layer to the neutral layer, $F \sin (\omega t)$ is the load, $I_{z}$ is inertia moment, $E$ is elastic modular.

\section{Preparation of transmission electron microscope (TEM) observation sample}

It was calculated that direction of the shear stress inside the bent beam is identical to the load, so a slice $(6 \mathrm{~mm} \times 6 \mathrm{~mm} \times 0.5 \mathrm{~mm})$ was cut via wire electrical discharge machining at the position in the shear stress action face, it is shown in Fig. 2, the point $\mathrm{O}$ is the center of the sample. 


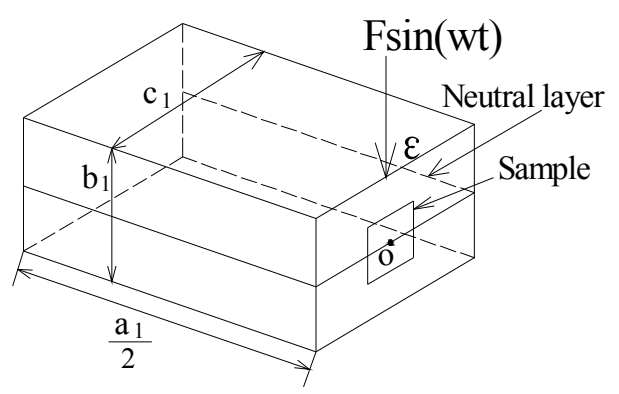

Fig. 2. Position of TEM observation sample

The shear stress value in neutral layer is the largest in the rectangular bending beam, calculation of the largest shear stress at point $\mathrm{O}$ is as follows.

$$
\tau_{\max }=\frac{E b_{1}}{2 a_{1}} \varepsilon
$$

Finally, TEM foil was prepared by ion-thinning after mechanical grinding from the slice sample. The diameter of the TEM foil is $3 \mathrm{~mm}$, the Point $\mathrm{O}$ in Fig. 2 is the center of the TEM foil. The observation area was around the point $\mathrm{O}$.

\section{Results and discussion}

\section{Observation of dislocation}

The dislocation images were observed by TEM. Fig. 3 shows that dislocation source has been slipped and multiplied, it has formed many dislocation loops. In Fig. 3a and Fig. 3b, it can be seen that the dislocations multiplied and extended uniformly, specifically, many dislocation loops in a hemicycle multiplied and extended uniformly in Fig. 3b. The result is the dislocation density increases obviously when the beam was been cyclic bending.

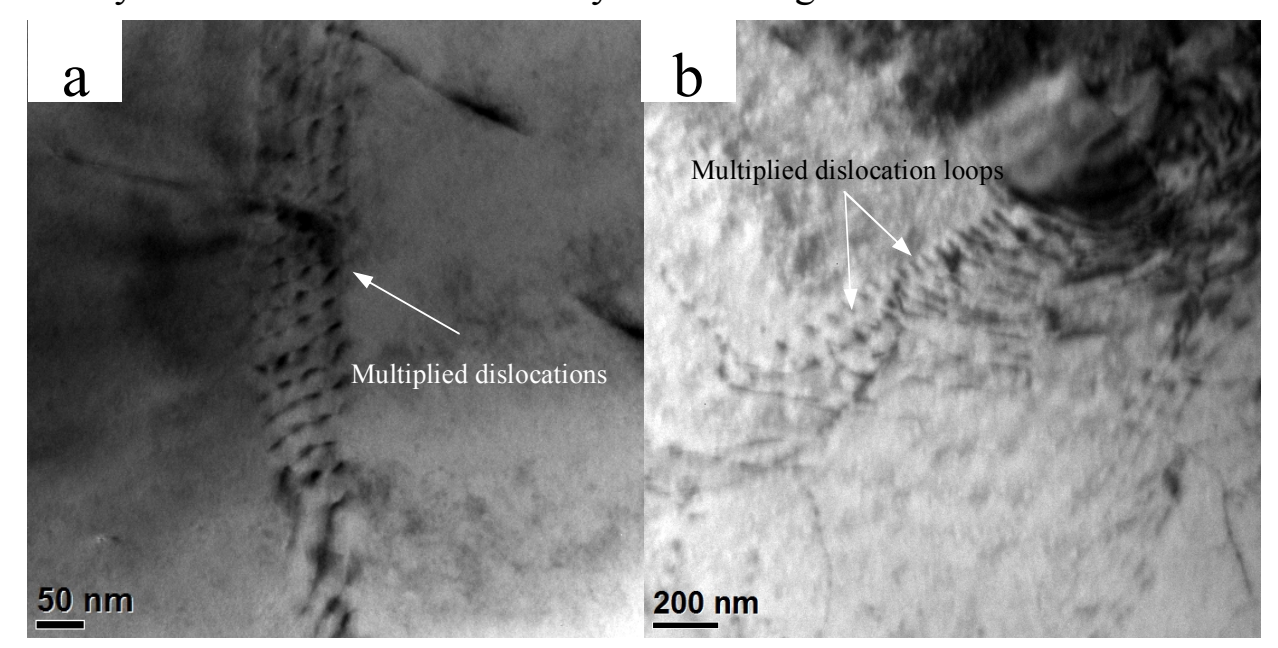

Fig. 3 The images of dislocation slipping and multiplication

Form the above dislocation images, it can be found that the dislocation multiplication behavior has taken place, and the dislocation multiplication accords with F-R source mechanism, The process of dislocation multiplication caused by shear stress is shown in Fig. 4. The result is the dislocation number and the dislocation density in AZ61 magnesium alloy increase significantly with the increase of stain amplitude. 


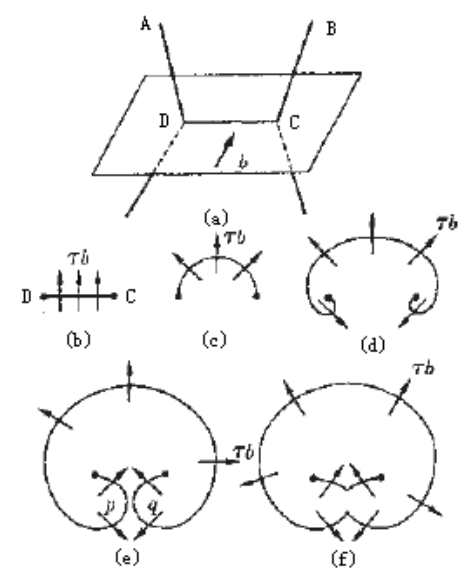

Fig. 4 Frank-Read source mechanism and process [6]

\section{Conclusions}

Summarizing the detailed research that we have carried out on the dislocation multiplication behavior of AZ61 magnesium alloy in condition of cyclic bending vibration, we could come to the following conclusions:

(1) The dislocation multiplication caused by the shear stress accords with Frank-Read source mechanism.

(2) The process of dislocation multiplication behavior contains dislocation slipping, dislocation multiplication and dislocation extension.

(3) The mobility dislocation number and the mobility dislocation density in AZ61 magnesium alloy increase significantly with the increase of stain amplitude.

The results are important foundations of researching on the dislocation damping mechanism and the change of mechanical properties of magnesium alloys.

\section{Acknowledgments}

The authors would like to acknowledge National Natural Science Foundation of China (Grant No. $51175533)$.

\section{References}

[1] K. Sugimoto, K. Niiya, T. Okamoto, K. Kishitake. A study of damping capacity in Magnesium alloys. Trans. Jpn. Inst.Met. 18 (1977) 277-288.

[2] Chuming LIU, Renfeng JI, Haitao ZHOU, Mingan CHEN. Research and development progress of damping capacity of magnesium and Magnesium alloys. The Chinese Journal of Nonferrous Metals. 15 (2005) 1319-1325.

[3] Nishiyama N, Asano S. Evaluation of amplitude dependence of internal friction in high-damping metals. J Jpn Inst Met. 65 (2001) 109-114.

[4] Z. Trojanová, A. Mielczarek b, W. Riehemann b, P. Lukáč. Cyclic bending and the damping behaviour of short fibre-reinforced Magnesium alloy AZ91. Composites Science and Technology. 66 (2006) 585-590.

[5] Di-Qing Wan. Strain amplitude-dependent internal friction of as-cast high damping Magnesium alloy during cyclic vibration. Rare Met. 32 (2013) 25-28.

[6] Kuanfu HA, Micro-theory of Metal Mechanical Properties, Science Press, Beijing 1983. 